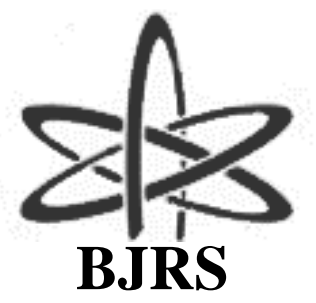

\author{
BRAZILIAN JOURNAL \\ $\mathrm{OF}$ \\ RADIATION SCIENCES \\ 06-02-B (2018) 01-12
}

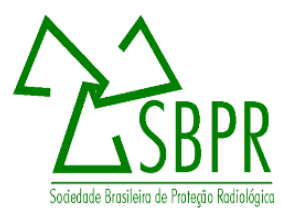

\title{
Avaliação dos parâmetros de aquisição de imagens SPECT para Ítrio-90 em radiosinoviortese
}

\author{
B. C. de Toledo ; F. A. Coelho $;$; S. M. Ramos ; S. Thomas $;$; S. A. de Souza ${ }^{\text {b }}$; M.
}

A. Pinheiro ${ }^{\text {; }}$ L. V. de Sáa

${ }^{a}$ Departamento de Física Médica, Instituto de Radioproteção e Dosimetria/ Comissão Nacional de Energia Nuclear, 22780-160, Rio de Janeiro-RJ, Brasil

${ }^{b}$ Departamento de Radiologia/Serviço de Medicina Nuclea, Hospital Universitário Clementino Fraga Filho/Universidade Federal do Rio de Janeiro, 21941-590, Rio de Janeiro-RJ, Brasil

bruce@bolsista.ird.gov.br

\section{RESUMO}

Introdução: A avaliação da distribuição de material radioativo na articulação por imagens SPECT pós radiosinoviortese (RSO) com ítrio-90 $\left({ }^{90} \mathrm{Y}\right)$ visa garantir a eficácia e segurança da terapia. Contudo, a qualidade de imagem por Bremsstrahlung possui grandes limitações, tornando necessários estudos sobre a otimização de parâmetros de aquisição SPECT e dos métodos de reconstrução de imagem para o radionuclídeo ${ }^{90} \mathrm{Y}$. Métodos: Foram obtidas imagens SPECT de um simulador de acrílico contendo quatro inserções cilíndricas de diferentes diâmetros que mimetizam lesões captantes, com colimadores de alta, média e baixa (HEGP, MEGP e LEHR) energia, janela de energia de $130 \mathrm{keV}$, largura $70 \%$ e matriz 64 x 64. Os métodos de reconstrução utilizados foram FBP e OSEM, com aplicação de diferentes filtros e número de iterações. Resultados: O maior contraste de imagem foi observado para o método de reconstrução iterativo, com a aplicação do filtro Gauss, sendo que o método FBP apresentou resultados insatisfatórios. O acréscimo no número de iterações no método OSEM não apresentou aumento da qualidade da imagem. Há menor discrepância entre os valores de captação das diferentes lesões para as aquisições com o colimador HEGP. Conclusões: Há diferença significativa na quantificação entre as reconstruções FBP e OSEM. O uso do colimador HEGP e do método de reconstrução iterativo OSEM com a aplicação do filtro Gauss se mostrou como o protocolo de aquisição de imagem mais indicado para estudos com ${ }^{90} \mathrm{Y}$.

Palavras-chave: Radiosinoviortese, SPECT, Ítrio-90.

ISSN: 2319-0612

Accepted 2018-07-31 


\begin{abstract}
Introduction: The evaluation of the radioactive material distribution in the articulation by SPECT images after radiosinoviortese (RSO) with yttrium-90 $\left({ }^{90} \mathrm{Y}\right)$ aims to guarantee the efficacy and safety of the therapy. However, Bremsstrahlung's image quality has major limitations, demanding studies on the optimization of SPECT acquisition parameters and image reconstruction methods for the ${ }^{90} \mathrm{Y}$ radionuclide. Methods: SPECT images were obtained from an acrylic simulator containing four cylindrical inserts of different diameters that mimic lesions by using high, medium and low collimators (HEGP, MEGP and LEHR) energy, with a $130 \mathrm{keV}$ energy window, $70 \%$ width and 64 x 64 matrix. The reconstruction methods tested were FBP and OSEM, with application of different filters and number of iterations. Results: The highest image contrast was observed for the iterative reconstruction method, with Gauss filter; FBP method presented unsatisfactory results. The increase in the number of iterations in OSEM method did not present an increase in image quality. There is less discrepancy between the uptake values of the different lesions for HEGP collimator acquisitions. Conclusions: There is a significant difference between FBP and OSEM reconstructions in quantification. The use of HEGP collimator and iterative reconstruction method OSEM with the application of the Gauss filter was shown as the most suitable image acquisition protocol for studies with ${ }^{90} \mathrm{Y}$.
\end{abstract}

Keywords: Radiosynoviorthesis, SPECT, Yttrium-90.

\title{
1. INTRODUÇÃO
}

A radiosinoviortese (RSO), também conhecida como sinovectomia radioisotópica, sinovectomia de radiação ou radiosinovectomia, é um tratamento que consiste na aplicação intraarticular de um radiofármaco beta-emissor específico com o objetivo de irradiar a membrana sinovial patológica que pode causar fibrose e reduzir a mobilidade articular [1].

A Associação Europeia de Medicina Nuclear (European Association on Nuclear Medicine$E A N M)$ recomenda o uso de ${ }^{90} \mathrm{Y}$-silicato/citrato, ${ }^{186} \mathrm{Re}$-sulfato ou ${ }^{169}$ Er-citrato para terapias de RSO [2]. A escolha do radionuclídeo ideal para o tratamento de articulações pequenas (dedos), médias (cotovelo, pulso e tornozelo) e grandes (quadril e joelho) depende da penetração das partículas $\beta$ do radionuclídeo escolhido e do tamanho de partícula coloidal, que deve corresponder à espessura da sinóvia inflamada [3]. Um radionuclídeo amplamente utilizado para a RSO na articulação do joelho é o ${ }^{90} \mathrm{Y}$, com eficácia estimada em 60 a $80 \%$ [2], apresentando dificuldades atribuídas a fatores relacionados ao paciente, como o tipo, curso e atividade inflamatória dentro da articulação [4, 5]. 
A hidroxiapatita [Ca10(PO4)6(OH)2], que é o fármaco utilizado no Brasil, apresenta boa resposta em RSO por ser o componente mais abundante da matriz óssea [6], possuir estabilidade bioquímica in vitro duradoura [7] e pela característica biodegradável mostrada em estudos com modelos animais [8].

A avaliação da distribuição e não extravasamento do material radioativo da articulação é um dos fatores que pode garantir a eficácia e segurança da RSO. Para isso, os pacientes podem realizar cintilografia de corpo inteiro, estática e tomográfica por SPECT (Single Photon Emission Tomography), 2 h e 48 ou 72 h após o procedimento [2]. Para o caso do uso de radionuclídeos emissores de partículas $\beta$ como, por exemplo, ${ }^{90} \mathrm{Y}$, a produção de fótons de Bremsstrahlung pode também ser visualizada por cintilografia [9]. Porém, as imagens obtidas por câmaras gama não são facilmente quantificáveis, apesar de fornecerem evidência de que há certo grau de padronização do procedimento, incluindo o local da injeção [10].

Embora tecnicamente viável, a qualidade de imagem para o ${ }^{90} \mathrm{Y}$ Bremsstrahlung é limitada pela sobrecarga de atenuação de tecidos, espalhamento de fótons internos, taxas de contagem variável de fótons bremsstrahlung emitidos, ampla gama de energia de fótons produzida, baixa resolução espacial, tipo de colimador utilizado e, ainda, processamento de imagem escolhido [11].

Os métodos de reconstrução existentes em equipamentos de geração de imagem em medicina nuclear são o analítico e o iterativo. O método analítico mais aplicado é a retroprojeção filtrada (Filtered Back Projection - FBP). Quanto ao método iterativo, o algoritmo mais utilizado é o OSEM (Ordered Subsets Expectation Maximization) [12]. Com a finalidade de corrigir distorções na imagem reconstruída são utilizados filtros que removem ruídos e melhoram a qualidade da imagem; estes são aplicados após a reconstrução e sua escolha depende do operador [13].

A proposta deste estudo foi analisar a influência dos diferentes parâmetros de aquisição e métodos de reconstrução tomográfica utilizados em exames por SPECT pós RSO com ${ }^{90} \mathrm{Y}$ Hidroxiapatita possibilitando melhor visualização da distribuição do material radioativo na articulação e possível quantificação.

\section{MATERIAIS E MÉTODOS}


Os ensaios foram realizados no Serviço de Medicina Nuclear do Hospital Universitário Clementino Fraga Filho (HUCFF), unidade da Universidade Federal do Rio de Janeiro (HUCFF/UFRJ), em parceria com o Instituto de Radioproteção e Dosimetria (IRD/CNEN).

\subsection{AQUISIÇÃO DAS IMAGENS}

A aquisição das imagens foi feita no equipamento SPECT GE Healthcare, modelo Millennium-MG. A câmara gama apresenta dois detectores com cristal de $\mathrm{NaI}(\mathrm{Tl})$ (Iodeto de Sódio dopado com tálio). Para definir o melhor colimador para aquisição de imagens de ${ }^{90} \mathrm{Y}$ foi realizada uma comparação das imagens obtidas com os três tipos disponíveis no serviço: Baixa Energia Alta Resolução (Low Energy High Resolution-LEHR); Média Energia Uso Geral (Medium Energy General Purpose-MEGP); e Alta Energia Uso Geral (High Energy General Purpose-HEGP). Foram feitas 64 projeções com tempo de 30 segundos por projeção, utilizando uma janela de energia centralizada em $130 \mathrm{keV}$ e largura de $70 \%$, e matriz de 64 x 64 .

\subsection{SIMULADOR}

Foi utilizado um simulador confeccionado pelo Instituto de Radioproteção e Dosimetria (IRD) de forma a representar o modelo Jaszczak SPECT/PET Flangeless sugerido pelo Colégio Americano de Radiologia (American College of Radiology-ACR). Este possui as seguintes dimensões: diâmetro interno $21,6 \mathrm{~cm}$, altura $18,6 \mathrm{~cm}$, espessura $3,2 \mathrm{~cm}$ e tampa contendo sete cilindros com altura de $38,1 \mathrm{~mm}$, sendo quatro cilindros de plástico para simulação de lesões captantes ("quentes") com diâmetros de 8,0 mm (2 mL); 12,0 mm (4,5 mL); 16,0 mm (8 mL) e 25,0 mm (19 mL); dois cilindros de plástico para simulação de ar e água, com diâmetro de 25,0 mm; e um cilindro de teflon para simulação do tecido ósseo, com diâmetro de 25,0 mm.

O simulador foi preparado com os cilindros preenchidos com solução de concentração em atividade $0,74 \mathrm{MBq} / \mathrm{ml}$ retratando a concentração considerada em um tratamento de RSO com injeção de $185 \mathrm{MBq}$ de atividade para um volume de $250 \mathrm{~cm}^{3}$ [2, 14, 15]; o corpo do simulador foi preenchido com água. As medidas de atividades foram realizadas no medidor de atividade disponível no serviço, de fabricação da Capintec - Modelo CRC-25-R - N/S: 251200. 


\subsection{MÉTODOS DE RECONSTRUÇÃO}

Para cada tipo de aquisição foram feitas reconstruções com os métodos FBP e iterativo. O FBP, método mais rápido de todas as técnicas de reconstrução, consiste basicamente na filtragem das projeções no espaço de frequências, usando o filtro ramp e, após, a realização da transformada inversa retroprojeta esses valores para formação da imagem final [16].

O método iterativo inicia o processo de reconstrução com a estimativa de uma imagem inicial que, normalmente, é uma distribuição de atividade uniforme. A seguir, um conjunto de dados de projeção é estimado a partir dessa imagem inicial, utilizando um processo matemático denominado de projeção seguinte (forward projection). As projeções resultantes são comparadas com as projeções originais, e as diferenças entre ambas são utilizadas para atualizar a nova imagem, a partir de um critério de semelhança [17].

As imagens obtidas neste estudo foram reconstruídas utilizando o método FBP com filtros Metz, Butterworth e Hann. Para as reconstruções pelo método iterativo foram utilizadas diferentes iterações $(3,5,8$ e 10) e 8 subconjuntos, seguido da aplicação de filtros Butterworth, Hann e Gauss.

\subsection{ANÁLISE DAS IMAGENS}

As imagens em formato DICOM (Digital Imaging and Communications in Medicine) foram transferidas para outro computador e analisadas quanto à qualidade pelo software RadiAnt ${ }^{\circledR}$ que fornece a média de contagens por pixel de cada região de interesse (Region of Interest, ROI), tendo como guia a Norma IEC 61675-2, da Comissão Eletrotécnica Internacional (International Electrotechnical Commission - IEC) que aborda a avaliação da qualidade da imagem em medicina nuclear [18]. As propriedades avaliadas neste estudo foram as que se apresentam como críticas para a diferenciação de regiões captantes ou não captantes, tais como contraste e relação contraste-ruído, dadas pelas equações: 
Variação do ruído

$$
C N j=S j / B j
$$

Onde, Sj é o desvio padrão dos valores de radiação de fundo (background - BG) medidos com ROI de mesmas dimensões das esferas ou cilindros captantes $j$; $B j$ é a média dos valores medidos de $\mathrm{BG}$ em regiões de interesse com as mesmas dimensões da esfera ou cilindro captante $j$.

Contraste

$$
\text { Contraste }=P j / B j
$$

Onde $P j$ é a média dos valores encontrados na ROI da lesão captante $j$; e $B j$ é a média dos valores encontrados de BG medidos com o mesmo tamanho de ROI correspondente a lesão $j$.

Razão contraste-ruído (contrast-to-noise ratio, CNR)

$$
C N R j=(P j / B j-1) / C N j
$$

Onde $P j$ é o valor médio encontrado na ROI da lesão captante $j ; B j$ é a média dos valores de BG encontrados na ROI referente à $j$; $C N j$ é o coeficiente de variação de ruído para a lesão $j$.

\section{RESULTADOS E DISCUSSÕES}

Foram gerados 45 resultados para as diferentes combinações utilizadas na aquisição e reconstrução das imagens. Destes, foram escolhidos 5 resultados considerados melhores para cada aquisição (colimador LEHR, MEGP e HEGP) e reconstrução (FBP e OSEM) considerando os requisitos: maior contraste, maior razão contraste-ruído e menor diferença entre os valores encontrados nas ROI de diâmetros distintos. A figura 1 mostra os resultados separados por tipo de colimador utilizado na aquisição, separados por códigos definidos da seguinte forma:

Colimador $(1=L E H R, 2=M E G P$ ou $3=H E G P)+\operatorname{Reconstrução~}(\boldsymbol{a}=F B P, \boldsymbol{b}=\operatorname{OSEM}(3 i), \boldsymbol{c}=$ $\operatorname{OSEM}(5 i), \boldsymbol{d}=\operatorname{OSEM}(8 i)$ ou $\boldsymbol{e}=\operatorname{OSEM}(10 i))+$ Filtro $(B=$ Butterwoth, M=Metz ou G= Gauss). Exemplo: $2 \mathrm{aM}=$ Colimador MEGP, método FBP com filtro Metz. 
Figura 1: Contraste e da Razão Contraste-Ruído.

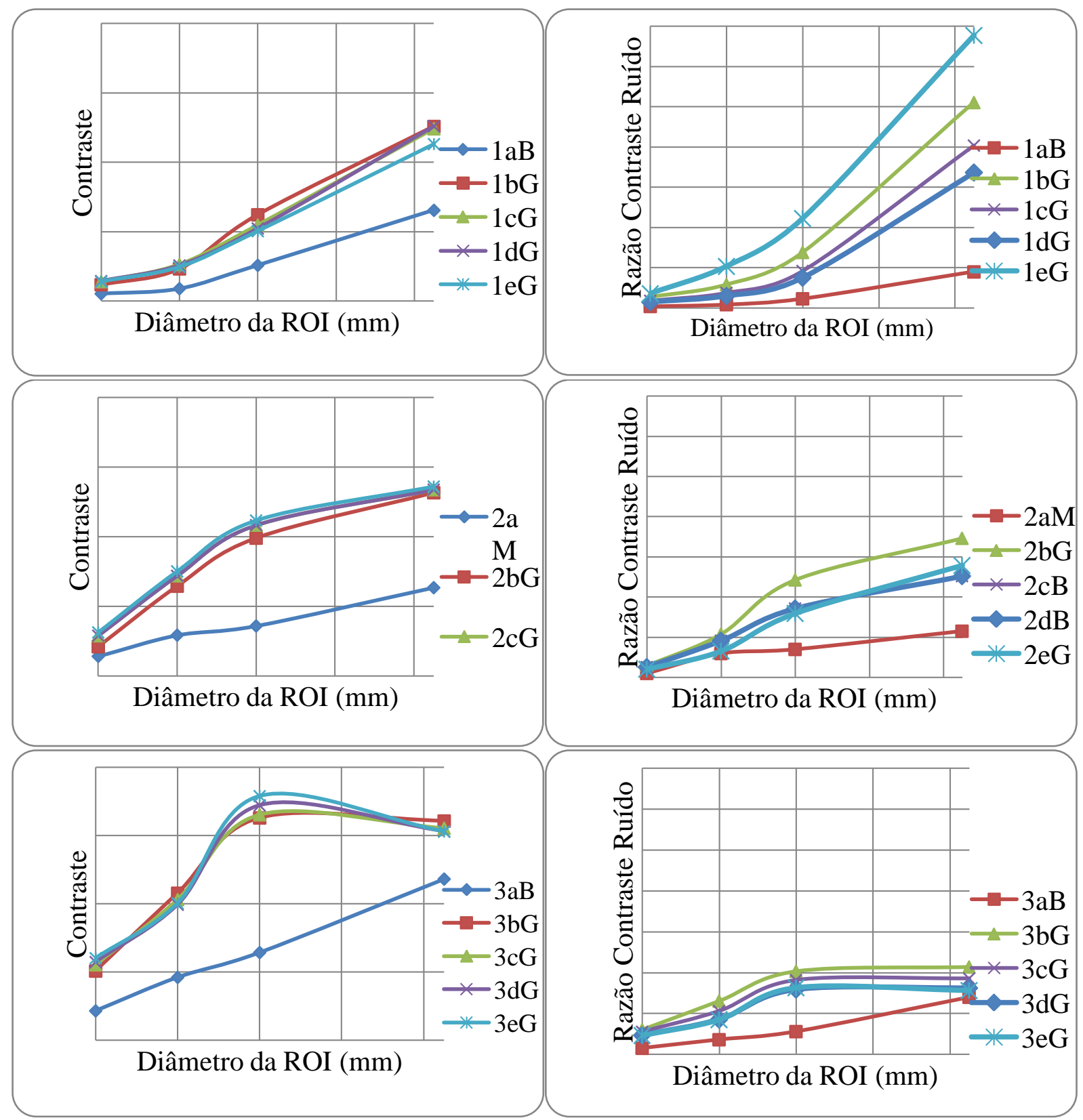

A partir dos gráficos apresentados na figura 1 é possível observar que para se obter maior contraste de imagem, o método de reconstrução iterativo com a aplicação do filtro Gauss é o mais indicado para aquisições feitas com os três tipos de colimadores. Enquanto, o método FBP apresenta resultados insatisfatórios para todas as reconstruções, mesmo com uso de diferentes filtros. 
Os valores obtidos para as aquisições com o colimador LEHR mostram que há superestimativa dos valores de contraste em ROI com diâmetro acima de $16 \mathrm{~mm}$. Logo, os colimadores MEGP e HEGP costumam ser mais indicados para aquisições de imagens ${ }^{90} \mathrm{Y}$, justificando o uso destes em estudos preliminares [11].

A Razão Contraste-ruído, que representa a propriedade mais utilizada na avaliação de imagens médicas [18], mostrou valores similares aos encontrados para o contraste, confirmando a superioridade dos resultados para aquisições feitas com os colimadores MEGP e HEGP reconstruídas com o método iterativo. Porém, para essa propriedade, o uso do filtro Butterwoth também se mostrou eficiente em aquisições feitas com o colimador MEGP.

O acréscimo de iterações em reconstruções pelo método iterativo OSEM não apresentou aumento da qualidade da imagem, uma vez que os melhores resultados variaram entre 3 a 10 iterações. Com isso, o ajuste deste recurso deve ser realizado cuidadosamente para a finalidade desejada em estudos com ${ }^{90}$ Y. Neste estudo, 3 iterações mostraram-se eficiente para se obter uma boa imagem.

A figura 2 apresenta as melhores imagens, utilizando a propriedade Razão Contraste-ruído como critério de avaliação, para cada tipo de colimador.

Figura 2: Imagens do simulador ACR adquiridas com os três tipos de colimador.

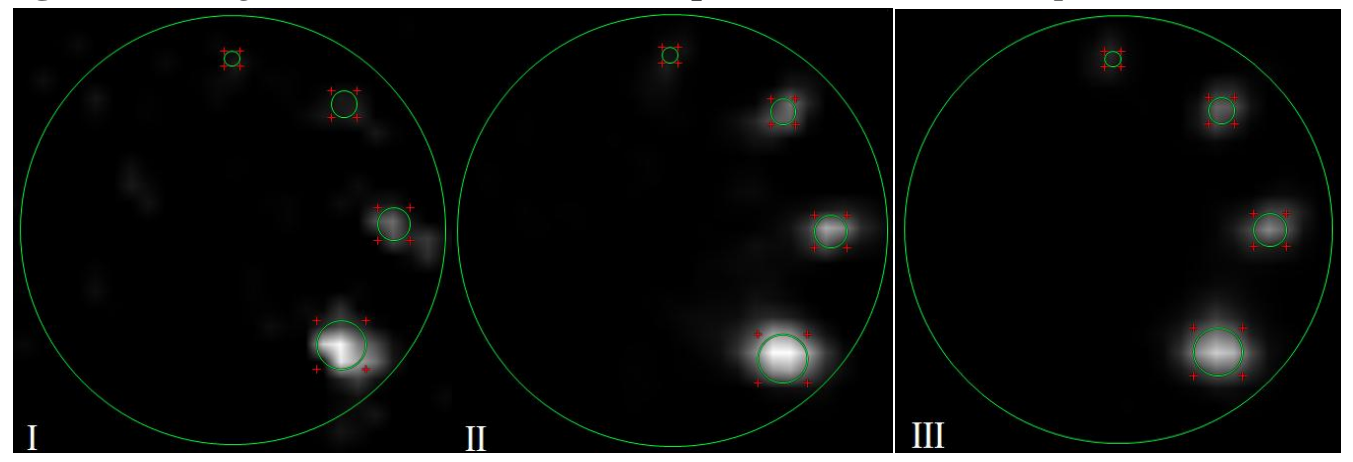

Por comparação é possível observar que a aquisição feita com o colimador I (LEHR) é a que apresenta maior ruído, mostrando que seu uso não é adequado para aquisições pós RSO com ${ }^{90} \mathrm{Y}$, pois pode apresentar regiões quentes, onde não há material radioativo, levando o médico a uma avaliação errônea da distribuição do radiofármaco na articulação do paciente e a um possível prognóstico incorreto do tratamento. 
Houve maior sensibilidade quando o colimador LEHR foi usado, porém a degradação da imagem devido a grande quantidade de ruído foi crítica. Embora a sensibilidade do colimador MEGP seja ligeiramente maior à do colimador HEGP, foi possível observar que a diferença de captação entre ROIs de diferentes diâmetros não apresentam discrepância (figura 1 - Razão Contraste-ruído-HEGP), o que é o resultado adequado.

A influência da aplicação de filtros na reconstrução da imagem pode ser vista na tabela 1 , que mostra os valores de contraste e razão contraste-ruído para o melhor parâmetro de aquisição e reconstrução de imagem encontrada neste estudo (Colimador HEGP + Reconstrução OSEM com 3 iterações).

Tabela 1: Valores de contraste e razão contraste-ruído na aplicação de diferentes filtros para as aquisições feitas com o colimador HEGP e reconstruídas com 3 iterações.

\begin{tabular}{ccccccc} 
& \multicolumn{3}{c}{ Contraste } & \multicolumn{3}{c}{ Razão Contraste-Ruído } \\
\cline { 2 - 7 } $\begin{array}{c}\text { ROI } \\
(\mathbf{m m})\end{array}$ & Butterwoth & Hann & Gauss & Butterwoth & Hann & Gauss \\
\hline $\mathbf{2 5}$ & 60,9 & 56,8 & 64,3 & 113,3 & 104,2 & 107,2 \\
$\mathbf{1 6}$ & 50,9 & 47,4 & 65,2 & 91,4 & 86,4 & 102,0 \\
$\mathbf{1 2}$ & 35,7 & 32,6 & 43,1 & 61,4 & 58,5 & 65,6 \\
$\mathbf{8}$ & 14,1 & 13,4 & 20,3 & 24,3 & 23,4 & 30,5 \\
\hline
\end{tabular}

O uso do filtro Gauss apresentou o resultado mais esperado, pois a diferença entre ROIs de diferentes tamanhos é menor, quando comparada aos resultados com o uso de outro filtro, e também, maior sensibilidade para lesões de pequenos diâmetros. O filtro Hann gerou imagens com menor contraste e razão contraste-ruído quando comparado aos outros dois filtros, não sendo um filtro indicado para estudos $\operatorname{com}^{90} \mathrm{Y}$.

Existem poucos estudos com o objetivo de avaliar a qualidade da imagem obtida pós RSO, como as por Bremsstrahlung quando utilizado o radionuclídeo ${ }^{90} \mathrm{Y}$. Além disso, dentre os poucos estudos que avaliaram a aquisição ou o tipo de reconstrução, a maioria não chegou a uma conclusão absoluta de qual método seria mais indicado, como foi feito neste trabalho. Ainda assim, nenhum deles investigou uma variedade de valores das possíveis combinações como o presente estudo [11]. 


\section{CONCLUSÕES}

Os resultados obtidos corroboram alguns estudos sobre parâmetros para aquisição de imagens de ${ }^{90} \mathrm{Y}$ em SPECT descritos na literatura, relacionados ao uso de diferentes colimadores, em que é possível observar que os colimadores MEGP e HEGP são mais adequados para uso clínico.

Os métodos de reconstrução de imagens têm importância relevante na qualidade da imagem, mostrando diferença significativa entre as reconstruções FBP e OSEM, onde se pode observar que os resultados pela FBP são inferiores, tornando o uso de reconstruções iterativas (OSEM) mais adequado para estudos clínicos com ${ }^{90} \mathrm{Y}$.

O uso do colimador HEGP na aquisição da imagem e do método de reconstrução iterativo OSEM com a aplicação do filtro Gauss foi o protocolo de aquisição de imagem mais indicado para estudos da distribuição do material radioativo na articulação pós RSO, pois apresenta menor ruído, maior contraste e menor discrepância na captação entre lesões de diferentes tamanhos.

\section{REFERÊNCIAS}

[1]. SCHNEIDER, P.; FARAHATI, J.; REINERS C. Radiosynovectomy in rheumatology, orthopedics, and hemophilia. J Nucl Med, v. 46 Suppl 1, p. 48-54, 2005.

[2]. CLUNIE, G.; FISCHER, M. EANM procedure guide lines for radiosynovectomy. Eur J Nucl Med Mol Imaging, v. 30(3), p. 12-16, 2003.

[3]. JANKOVIC, D.; VRANJES-DJURIC, S.; DJOKIC, D.; et al. 90Y-labeled tin fluoride colloid as a promising therapeutic agent: preparation, characterization, and biological study in rats. J Pharm Sci. v. 101(6), p. 2194-2203, 2012.

[4]. HEUFT-DORENBOSCH, LL.; DE VET, HC.; VAN DER LINDEN, S. Yttrium radiosynoviorthesis in the treatment of knee arthritis in rheumatoid arthritis: a systematic review. Ann Rheum Dis. v. 59(8), p. 583-586, 2000. 
[5]. PIRICH, C.; SCHWAMEIS, E.; BERNECKER, P.; et al. Influence of radiation synovectomy on articular cartilage, synovial thickness and enhancement as evidenced by MRI in patients with chronic synovitis. JNucl Med Off PublSocNucl Med. v. 40(8), p. 1277-1284, 1999.

[6]. SIEGAL, H.J.; LUCK, J.V.; SIEGEL, M.E.; et al. Hemarthrosis and synovitis associated with hemophilia: clinical use of P-32 chromic phospate synoviorthesis for treat-ment. Radiology. v. 190(1), p. 257-261, 1994.

[7]. CHINOL, M.; VAllabHAJOSUlA, S.; GOLDSMITH, S.J.; KLEIN, M.J.; et al. Chemistry and biological behavior of samarium-153 and rhenium-186-labeled hydoxyapa-tite partciles: potencial radiopharmaceuticals for radiation synovectomy. J Nucl Med. v. 34(9) p. 1536-1542, 1993.

[8]. SHORTKROFF, S.; MAHMOOD, A.; SLEDGE, C.B.; et al. Studies on Ho-166-labeled hydroxyapatite: a new agent for radiation synovectomy (abstract). J Nucl Med. v. 33, p. 937, 1992.

[9]. SMITHT; CRAWLEY, J. C. W.; SHAWE, D. J.; GUMPEL, J. M. SPECT using Bremsstrahlung to quantify $90 \mathrm{Y}$ uptake in Baker's cysts: its application in radiation synovectomy of the knee," European Journal of Nuclear Medicine, v. 14(9-10), p. 498-503, 1988.

[10]. JAHANGIER, Z.N.; VAN DER ZANT, F.M.; JACOBS, J.W.; et al. The intra-articular distribution of 90yttrium does not influence the clinical outcome of radiation synovectomy of the knee. AnnRheumDis. v. 66(8), p. 1110-1112, 2007.

[11]. WRIGHT C. L.; ZHANG, J.; TWEEDLEM, F.; et al. Theranostic Imaging of Yttium-90, Hindawi, v. 2015. 
[12]. POWSNER R. A., POWSNER E. R., Essential Nuclear Medicine Physics. BlackwellPublishing. Second edition, 2006.

[13]. MARQUES FILHO, O.; VIEIRA NETO, H.; Processamento Digital de Imagens. Brasport, Rio de Janeiro, 1999.

[14]. JOHNSON, L.S.; YANCH, J.C.; SHORTKROFF, S.; et al. Beta-particle dosimetry in radiation synovectomy. Eur J Nucl Med. v. 22(9), p. 977-988, 1995.

[15]. JOHNSON, L.S.; YANCH, J.C.; Calculation of beta dosimetry in radiation synovectomy using Monte Carlo simulation (EGS4). Med Phys. v. 20(3), p. 747-754, 1993.

[16]. Projeto FRIDA. Desenvolvimento de Plugin Java para reconstrução e visualização em Medicina Nuclear. Porto Alegre: 2005-2006;

[17]. LYRA, M.; PLOUSSI, A. Filtering in SPECT Image Reconstruction. International Journal of Biomedical Imaging. v. x, p. 1-14, 2011.

[18]. IEC- International Electrotechnical Commission - Radionuclide imaging devices Characteristics and test conditions - Part 2: Gamma cameras for planar, wholebody, and SPECT imaging, IEC 61675-2:2015. Edition 2.0, 2015. 